\title{
The impact of unrestricted access to direct-acting antiviral among incarcerated hepatitis $C$ virus-infected patients
}

Yu Jun Wong ${ }^{1,2,3}$, Prem Harichander Thurairajah ${ }^{4}$, Rahul Kumar ${ }^{1,3}$, Kwong Ming Fock ${ }^{1,2,3}$, Ngai Moh Law ${ }^{1,2}$, Sin-Yoong Chong', Fria Gloriba Manejero', Tiing-Leong Ang ${ }^{1,2,3}$, Eng Kiong Teo ${ }^{1,2,3}$, and Jessica Tan ${ }^{1}$

${ }^{1}$ Department of Gastroenterology and Hepatology, Changi General Hospital; ${ }^{2}$ Yong Loo Lin School of Medicine, National University of Singapore; ${ }^{3}$ Duke-NUS Medical School; ${ }^{4}$ National University Hospital, Singapore

\section{Graphical Abstract}

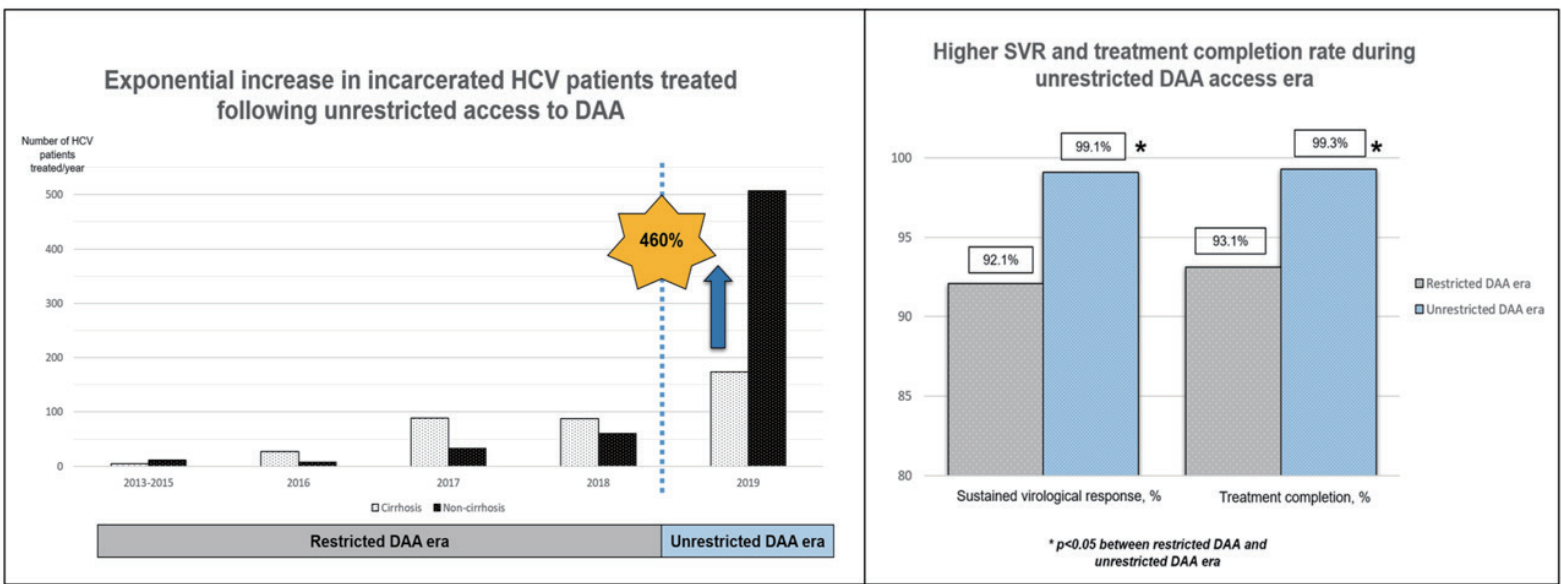

\section{Abbreviations:}

CGH, Changi General Hospital; Cl, confidence interval; DAA, direct-acting antiviral; Fib-4, fibrosis-4 index; GT, genotype; HBV, hepatitis B virus; HCV,

hepatitis C virus; HIV, human immunodeficiency virus; ITT, intention-to-treat; LSM, liver stiffness measurement; PP, per-protocol; PR, pegylated interferon and ribavirin; SOF, sofosbuvir; SVR, sustained virological response; VEL, velpatasvir
Corresponding author: Yu Jun Wong

Department of Gastroenterology and Hepatology, Changi General Hospital, 2 Simei Street 3 529889, Singapore

Tel: +65-69365729

E-mail: eugene.wong.y.j@singhealth.com.sg https://orcid.org/0000-0002-0727-1183 
Background/Aims: Despite the disproportionally high prevalence rates of hepatitis C virus (HCV) amongst the incarcerated population, eradication remains challenging due to logistic and financial barriers. Although treatment prioritization based on disease severity is commonly practiced, the efficacy of such approach remained uncertain. We aimed to compare the impact of unrestricted access to direct-acting antiviral (DAA) among incarcerated HCV-infected patients in Singapore.

Methods: In this retrospective study, we reviewed all incarcerated HCV-infected patients treated in our hospital during the restricted DAA era (2013-2018) and unrestricted DAA access era (2019). Study outcomes included the rate of sustained virological response (SVR), treatment completion and treatment default. Subgroup analysis was performed based on the presence of liver cirrhosis, HCV genotype and HCV treatment types.

Results: A total of 1,001 HCV patients was followed-up for 1,489 person-year. They were predominantly male (93\%) with genotype-3 HCV infection (71\%), and 38\% were cirrhotic. The overall SVR during the restricted DAA access era and unrestricted DAA access era were $92.1 \%$ and $99.1 \%$, respectively. Unrestricted access to DAA exponentially improved the treatment access among HCV-infected patients by $460 \%$, resulting in a higher SVR rate ( $99 \%$ vs. $92 \%, P=0.003)$, higher treatment completion rate $(99 \%$ vs. $93 \%, P<0.001)$ and lower treatment default rate $(1 \%$ vs. $9 \%, P<0.001)$.

Conclusion: In this large cohort of incarcerated HCV-infected patients, we demonstrated that unrestricted access to DAA is an impactful strategy to allow rapid treatment up-scale in HCV micro-elimination. (Clin Mol Hepatol 2021;27:474485)

Keywords: Prisons; Antiviral agents; Hepatitis C, Chronic

Study Highlights

1. Among incarcerated HCV-infected Asian patients, unrestricted access to DAA results in rapid treatment scale-up, more treatment completion, less treatment default and a higher SVR.

2. DAA results in high treatment success among incarcerated GT3 HCV-infected patients in the real-world setting.

\section{INTRODUCTION}

Hepatitis C virus (HCV) is a major health threat among persons who inject drugs and incarcerated population. ${ }^{1,2}$ Epidemiological data report a high prevalence of HCV among the incarcerated population when compared to the general populations. ${ }^{3}$ The global incarcerated population is estimated to be more than 10 million. ${ }^{4}$ In Singapore, the incarceration population rate per 100,000 is 195 and the conservative estimate of the incarcerated population is about 12,000 at any given time. ${ }^{5}$ The seroprevalence of $\mathrm{HCV}$ antibody among residents in correctional facilities is estimated to be as high as $31 \%{ }^{6}$ Incarcerated populations often engaged in high-risk behaviours such as intravenous drug abuse, unsafe sexual behaviour or tattoo practices, ${ }^{7,8}$ which put them at a high risk of HCV infection. ${ }^{9}$ As most of the prisoners will eventually be released, they could contribute to the community spread of HCV after released. ${ }^{10}$ Given the high prevalence of HCV among the incarcerated population, the World Health Organization had recommended prioritizing $\mathrm{HCV}$ treatment and eradication in this key population."

Incarceration provides access to a marginalized population in which HCV is prevalent, yet the access to healthcare is challenging upon release. In this regard, the treatment compliance during incarceration is anticipated to be better within a confined setting whereby treatment and follow-up are almost ensured. However, HCV treatment among the incarcerated population can also be challenging. ${ }^{12}$ Fundamentally, the prison had a complex role in prioritizing security over health. ${ }^{13}$ Frequent transfer between corrective facilities, short sentence time, early released on short notice could negatively impact on treatment compliance. ${ }^{14}$ Patients' factors such as treatment refusal due to low awareness and knowledge about HCV, co-existing mental illness as well as continuous exposure to violence and illicit drugs also affect HCV treatment uptake among the incarcerated HCV-infection patients. ${ }^{15}$ Physicians may defer treatment among the incarcerated HCV-infection patients because of the concern of ongoing highrisk behaviour leading to reinfection following sustained virological response (SVR). 
Introduction of pan-genotypic, highly efficacious, direct-acting antiviral (DAA) has given hope to eliminate HCV among the incarcerated population. DAA were available in Singapore since the year 2016. However, access to DAA was restricted due to the exorbitant cost and lack of subsidies for DAA treatment. ${ }^{16}$ Patients with urgent treatment indications such as decompensated liver cirrhosis, pegylated-interferon failure or pegylated-interferon intolerant, were prioritized for DAA treatment. 2019 was an important landmark for HCV treatment in Singapore as the price of the pan-genotypic DAA sofosbuvir (SOF)/velpatasvir (VEL) was revised. Furthermore, the government also provided financial subsidy for patients requiring HCV treatment in Singapore. ${ }^{17}$ These measures had collectively lifted the financial barriers of using DAA for HCV treatment in Singapore.

Despite the emerging DAA data among the incarcerated-HCV cohort, the DAA treatment outcomes from Asia, particularly South East Asia region, remained limited. ${ }^{18-24}$ As incarcerated HCV patients were generally excluded from clinical trial, real-world treatment outcome is valuable to strategize micro-elimination among the incarcerated population. Unfortunately, the real-world treatment outcomes from large-scale prison programmes from Asia remained scarce. ${ }^{19,22}$ Despite disproportionately high burden of HCV among the incarcerated population, treatment access remained restricted in these patients. Restricted treatment access among high HCV-prevalence group is an important barrier to HCV elimination. Therefore, this study aims to determine the impact of unrestricted access to DAA among incarcerated HCV patients. We hypothesized that the unrestricted access to DAA would increase the number of treated HCV patients, treatment compliance and outcomes among incarcerated HCV patients. To our best knowledge, this is the largest study reporting the real-world treatment outcome among incarcerated HCV patients in Asia.

\section{MATERIALS AND METHODS}

\section{Study design}

This is a retrospective cohort study of prospectively collected data on all incarcerated patients with chronic hepatitis $C$ who were treated in the Department of Gastroenterology \& Hepatology, Changi General Hospital (CGH) from 1st January 2013 to 31th December 2019. CGH is the main public hospital to provide medical care for the incarcerated population in Singapore. All HCV patients treated were prospectively included in a treatment database since 2013, as described in our previous study. ${ }^{25}$ Our health service research officer conducted an additional comprehensive search on all HCV patients using pharmacy prescription database in order to ensure all HCV patients treated were included. The study was performed following the Ethical standard of the Declaration of Helsinki. The Singhealth Institutional Review Board approved the study and granted consent waiver as this is a clinical audit of treatment outcomes.

\section{Study population}

The current study included all incarcerated HCV patients treated in our institution. Patients were monitored in a dedicated clinic following international guidelines. ${ }^{26,27}$ Liver cirrhosis was defined based on histological, radiological and clinical findings, which included liver stiffness measurement (LSM) $\geq 12 \mathrm{kPa} .{ }^{25}$ In subjects without clinical or radiological evidence of liver cirrhosis, non-invasive assessment of fibrosis stage was performed using fibrosis-4 index (Fib-4) and transient elastography (Fibroscan; Echosens, Paris, France), followed by liver biopsy in patients with discordant non-invasive fibrosis assessment. ${ }^{27}$ We measured serum HCV RNA levels using real-time polymerase chain reaction assay (Roche COBAS AmpliPrep/TaqMan version 2.0; Roche Molecular System, Branchburg, NJ, USA) which has the lowest detection limit of 12 IU/mL. SVR was defined as undetectable HCV RNA at 12 weeks following the completion of DAA, or 24 weeks following the completion of interferon-based therapy. ${ }^{26}$

\section{Care model}

In Singapore, the healthcare cost incurred during incarceration was borne by the state. Prisoners were offered an opt-in screening for HCV at first prison reception. Patients who were sero-positive for HCV or self-reported history of HCV were referred for a face-to-face consult in our institution to consider for HCV treatment. All incarcerated patients with detectable HCV RNA and adequate sentence duration to complete hepatitis $C$ treatment were offered HCV treatment during incarceration. All treatment decisions were at physician's discretion based on the presence of liver cirrhosis, co-infection with human immunodeficiency virus (HIV) or hepatitis B virus (HBV), comorbidities, and availability of treatment regimen. Patients with liver cirrhosis, prior treatment experience, co-infection with HIV and HBV infections were prioritized for HCV treatment during incarceration. To avoid treatment interruption, patients without adequate sentence period for treatment 
during incarceration were instructed to return for HCV treatment upon released back to the community. Patients who fulfilled urgent treatment indication were treated regardless of their remaining sentence periods. HCV patients treated with DAA were monitored for adverse events and SVR according to the clinical guidelines.

\section{Restricted versus unrestricted DAA era}

The access to DAA was restricted between 2013 and 2018 (hereafter known as restricted DAA access era) due to the unavailability and exorbitant cost of DAA treatment. Before introduction of DAA in 2016, all patients received interferon-based therapy for HCV treatment. Patients with genotype (GT) 3 received 24-weeks of pegylated interferon and ribavirin (PR) while patients with GT1 received 48-weeks of PR. Erythropoietin and filgrastim injection were given for patients with anemia or leukopenia during PR therapy. Following introduction of DAA in 2016, patients with contraindication to PR therapy such as severe renal impairment, advanced liver cirrhosis, autoimmune disease, significant psychiatric illness or having GT1 HCV infection received DAA as the first-line treatment. ${ }^{28}$ The access to DAA remained restricted among incarcerated GT3 patients whereby PR remained the first-line treatment, reserving DAA for patients who were cirrhotic or had treatment failure.

With unrestricted access to DAA in Singapore from 2019 (hereafter known as unrestricted DAA access era). DAA becomes the first-line treatment for all HCV patients, including the incarcerated populations, in Singapore. The options of DAA among incarcerated GT3 HCV patients included SOF/daclatasvir, SOF with PR or SOF/VEL for 12 weeks. The options of DAA among incarcerated GT1 HCV patients included ombitasvir/paritaprevir/ritonavir, SOF/ ledipasvir or SOF/VEL for 12 weeks. Ribavirin was added for HCV patients who with liver cirrhosis, prior treatment, clinically significant portal hypertension or stable hepatocellular carcinoma, as per physician's discretion. ${ }^{25}$

\section{Study outcomes}

The primary outcome was to compare the number of incarcerated-HCV patients treated and their SVR rates between restricted DAA access era and unrestricted DAA access era. For secondary outcomes, we compared the treatment completion rate and default rate among incarcerated HCV patients. To avoid selection bias, we reported SVR in both intention-to-treat (ITT) and per- protocol (PP) analysis, in percentages and $95 \%$ confidence interval (CI). In ITT analysis, we included all patients treated who showed SVR before our final data analysis (1st October 2020). Patients who were lost to follow-up or had early cessation of treatment due to any reasons such as adverse events or demised were included in ITT analysis. In PP analysis, we included all patients in ITT analysis who had completed treatment with available SVR results. ${ }^{25}$ The default rate was defined as the proportion of subjects without SVR results. Lastly, we performed subgroup analysis based on the presence of cirrhosis, HCV GT and types of HCV treatment.

\section{Statistical analysis}

Categorical variables such as the proportion of incarcerated HCV patients treated with DAA were compared using the chisquared test or Fisher's exact test when appropriate. We compared continuous variables using the Student's $t$-test or MannWhitney test depending on the distribution normality. A $P$-value of $<0.05$ was considered to be statistically significant. All statistical tests were performed using SPSS software version 23.0 (IBM Corporation, New York, NY, USA).

\section{RESULTS}

\section{Baseline characteristics}

A total of 1,001 incarcerated patients with detectable HCV RNA at baseline was treated. We summarized the baseline characteristics of this cohort in Table 1. The cohort was predominantly male $(92.7 \%)$ with a mean age of 50.3 years old. GT3 is the commonest (71.1\%) followed by GT1 (24.1\%). Total of 383 patients had liver cirrhosis. The diagnosis of liver cirrhosis was made based on histology or definite radiological features of cirrhosis $(n=248)$, $L S M \geq 12 \mathrm{kPa}$ with radiological features suggestive of cirrhosis ( $n=142$ ). In addition, a total of 35 patients had discordant results between LSM and radiological findings of cirrhosis and underwent liver biopsy. Among them, 14 were reclassified as liver cirrhosis while 21 were reclassified as non-cirrhotic. A total of 108 subjects had Fib-4 score below 1.45 and therefore did not underwent Fibroscan. Co-infection with HIV or HBV was found in 1\% and $1 \%$ of the cohort, respectively. Overall, the SVR rates for ITT and $\mathrm{PP}$ analysis were $93.6 \%(95 \% \mathrm{Cl}, 91.9-95.0 \%)$ and $97.0 \%$ (95\% Cl, 95.7-98.0\%), respectively (Fig. 1). The overall treatment 


\title{
CLINCAL and MOLECULAR
}

\author{
Volume_27 Number_3 July 2021
}

Table 1. Baseline characteristics of incarcerated HCV patients

\begin{tabular}{|c|c|c|c|c|}
\hline Variable & $\begin{array}{c}\text { Total } \\
(n=1,001)\end{array}$ & $\begin{array}{l}\text { Restricted DAA access } \\
\qquad(n=320)\end{array}$ & $\begin{array}{l}\text { Unrestricted DAA } \\
\text { access }(n=681)\end{array}$ & $P$-value* \\
\hline Age (years) & $50.3 \pm 9.6$ & $52.4 \pm 7.9$ & $49.3 \pm 10.2$ & $<0.001$ \\
\hline Gender & $928(92.7)$ & $303(94.7)$ & $625(91.8)$ & 0.061 \\
\hline HCV RNA (log IU/mL) & $5.9 \pm 0.9$ & $5.9 \pm 0.9$ & $6.0 \pm 0.9$ & 0.116 \\
\hline Genotype & & & & 0.370 \\
\hline 1 & $241(24.1)$ & $72(22.5)$ & $169(24.8)$ & \\
\hline 2 & $14(1.4)$ & $5(1.6)$ & $9(1.3)$ & \\
\hline 3 & $712(71.1)$ & $237(74.1)$ & $475(69.8)$ & \\
\hline 4 & $4(0.4)$ & 0 & $4(0.6)$ & \\
\hline 6 & $2(0.2)$ & 0 & $2(0.3)$ & \\
\hline Indeterminate & $28(2.8)$ & $6(1.9)$ & $22(3.2)$ & \\
\hline Fibrosis stage & & & & $<0.001$ \\
\hline F0/F1 & $231(23.1)$ & $11(3.4)$ & $220(32.3)$ & \\
\hline $\mathrm{F} 2$ & $94(9.4)$ & $12(3.8)$ & $82(12.0)$ & \\
\hline F3 & $185(18.5)$ & $81(25.3)$ & $104(15.3)$ & \\
\hline F4 & $383(38.2)$ & $209(65.3)$ & $174(25.6)$ & \\
\hline Not available & $108(10.8)$ & $7(2.2)$ & $101(14.8)$ & \\
\hline \multicolumn{5}{|c|}{ Cirrhosis-related complications } \\
\hline Ascites & $22(2.2)$ & $12(3.8)$ & $10(1.5)$ & 0.022 \\
\hline Esophageal varices & $66(6.6)$ & $37(11.6)$ & $29(4.3)$ & $<0.001$ \\
\hline Hepatic encephalopathy & $9(0.9)$ & $4(1.3)$ & $5(0.7)$ & 0.316 \\
\hline $\mathrm{HCC}$ & $14(1.4)$ & $4(1.3)$ & $10(1.5)$ & 0.520 \\
\hline \multicolumn{5}{|l|}{ Co-infection } \\
\hline HIV & $8(0.8)$ & $4(1.3)$ & $4(0.6)$ & 0.229 \\
\hline HBV & $11(1.1)$ & $4(1.3)$ & $7(1.0)$ & 0.486 \\
\hline \multicolumn{5}{|l|}{ Laboratory results } \\
\hline Albumin $(g / L)$ & $44 \pm 4$ & $43 \pm 5$ & $45 \pm 4$ & $<0.001$ \\
\hline Bilirubin (mmol/L) & $13.8 \pm 8.8$ & $15.7 \pm 8.2$ & $12.9 \pm 9.0$ & $<0.001$ \\
\hline Platelet $\left(10^{3} / \mathrm{uL}\right)$ & $198 \pm 70$ & $168 \pm 73$ & $212 \pm 64$ & $<0.001$ \\
\hline Sodium (mmol/L) & $140.2 \pm 2.6$ & $139.6 \pm 2.6$ & $140 \pm 2.6$ & $<0.001$ \\
\hline Creatinine (mmol/L) & $85.1 \pm 28.2$ & $86.5 \pm 39.1$ & $84.5 \pm 21.2$ & 0.293 \\
\hline PT (seconds) & $10.5 \pm 1.1$ & $10.8 \pm 1.4$ & $10.4 \pm 0.8$ & $<0.001$ \\
\hline DAA & $782(78.1)$ & $135(42.2)$ & $647(95.0)$ & $<0.001$ \\
\hline Complete treatment & $974(97.3)$ & $298(93.1)$ & $676(99.3)$ & $<0.001$ \\
\hline \multicolumn{5}{|l|}{ SVR; ITT } \\
\hline Not detected & 937 (93.6) & $268(83.8)$ & $669(98.2)$ & \\
\hline Detected & $29(2.9)$ & $23(7.2)$ & $6(0.9)$ & \\
\hline Defaulted & $35(3.5)$ & $29(9.1)$ & $6(0.9)$ & \\
\hline PP & $937(97.0)$ & $268(92.1)$ & $280(99.1)$ & 0.003 \\
\hline
\end{tabular}

Values are presented as mean \pm standard deviation or number (\%).

HCV, hepatitis C virus; DAA, direct-acting antivirals; HCC, hepatocellular carcinoma; HIV, human immunodeficiency virus; HBV, hepatitis B virus; PT, prothrombin time; SVR, sustained-virological response; ITT, intention-to-treat.

${ }^{*} P$-value comparison between restricted and unrestricted DAA access era. 


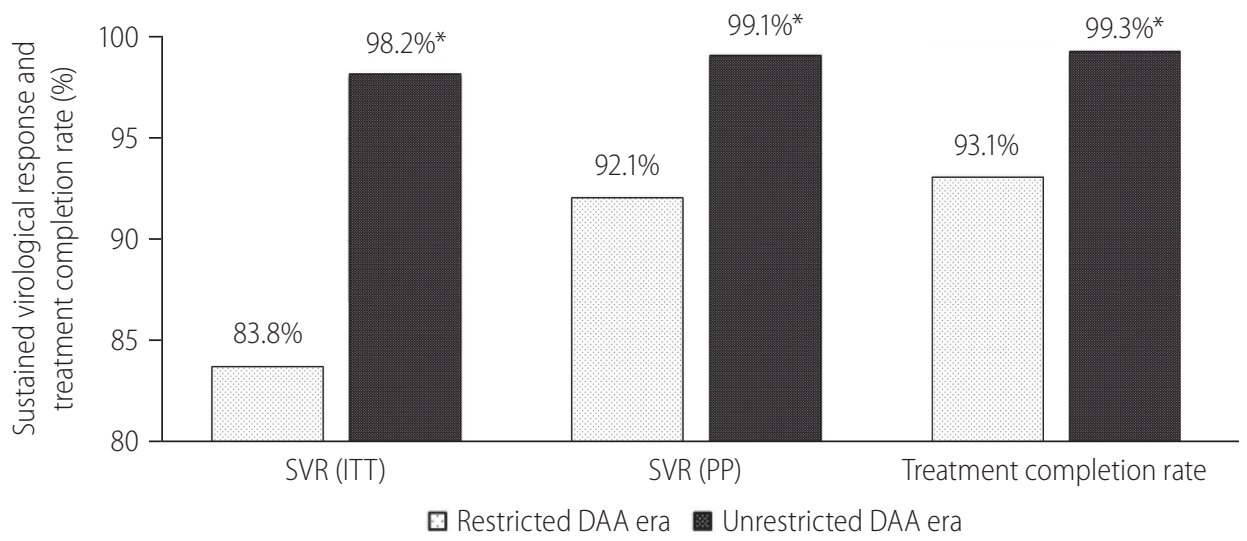

Figure 1. Treatment outcomes during restricted DAA access era versus unrestricted DAA access era. SVR, sustained-virological response; ITT, intention-to-treat; PP, per-protocol; DAA, direct-acting antivirals. ${ }^{*} P<0.05$ between restricted DAA and unrestricted DAA era.

Table 2. Treatment outcome (by year) among HCV-infected prisoners

\begin{tabular}{lcccccc}
\hline Year & $\begin{array}{c}\text { Number of } \\
\text { patients treated }\end{array}$ & $\begin{array}{c}\text { Liver cirrhosis } \\
(\mathbf{\%})\end{array}$ & $\begin{array}{c}\text { SVR rates (ITT) } \\
(\mathbf{\%})\end{array}$ & $\begin{array}{c}\text { SVR rates (PP) } \\
(\%)\end{array}$ & $\begin{array}{c}\text { Treatment } \\
\text { completion rate (\%) }\end{array}$ & $\begin{array}{c}\text { Default rate } \\
(\%)\end{array}$ \\
\hline 2013 & 6 & 16.7 & 83.3 & 83.3 & 100.0 & 0.0 \\
\hline 2014 & 2 & 0.0 & 100.0 & 100.0 & 100.0 & 0.0 \\
\hline 2015 & 8 & 50.0 & 87.5 & 87.5 & 87.5 & 0.0 \\
\hline 2016 & 34 & 79.4 & 85.3 & 93.5 & 94.1 & 8.8 \\
\hline 2017 & 122 & 73.0 & 84.4 & 91.2 & 92.6 & 7.4 \\
\hline 2018 & 148 & 59.5 & 82.4 & 93.1 & 93.2 & 11.5 \\
\hline 2019 & 681 & 25.6 & 98.2 & 99.1 & 99.3 & 0.9 \\
\hline
\end{tabular}

HCV, hepatitis C virus; SVR, sustained virological response; ITT, intention-to-treat; PP, per protocol.

completion and default rates were $97.3 \%$ and $3.5 \%$, respectively. HCV reinfection was not detected over the median follow-up of 1.2 years (interquartile range, $0.7-1.8$ years).

\section{Restricted DAA access era (2013-2018)}

Overall, 320 (32.0\%) HCV patients were treated during the restricted DAA era. Among them, 209 (65.3\%) had liver cirrhosis, and none defaulted PR therapy. The overall SVR rate during this period in ITT and PP protocol was $83.8 \%(95 \% \mathrm{Cl}, 79.2-87.6 \%)$ and $92.1 \%(95 \% \mathrm{Cl}, 88.4-94.9 \%)$, respectively (Table 1). The annual SVR rates ranged between $83.3 \%$ and $100 \%$ (Table 2).

Before DAA was introduced, only a total of 16 incarcerated HCV patients was treated with PR therapy between year 2013 and 2015. All patients had GT3 HCV infection, except for one, who had GT1 HCV infection.

When DAA was first introduced in Singapore in 2016, a total of
34 incarcerated HCV patients was treated. Compared to year 2015, introduction of DAA in 2016 allowed more patients to receive DAA (47\% vs. $0 \%, P=0.016$ ), more cirrhotic patients being treated ( $79 \%$ vs. $50 \%, P=0.170$ ) yet resulting in significantly higher SVR rates in PP analysis $(93.5 \%$ [95\% Cl, 78.6-99.2\%] vs. $87.5 \%$ [95\% Cl, 47.3-99.7\%]; $P=0.037$; Table 2).

\section{Unrestricted DAA access era (2019)}

Following the unrestricted access to DAA in 2019, the number of incarcerated HCV patients treated increased exponentially by $460 \%$ to 681 (Fig. 2). During the era of unrestricted DAA access, more patients received DAA ( $95 \%$ vs. $42 \%, P<0.001$ ), completed HCV treatment (99\% vs. 93\%, $P<0.001)$ and less patients defaulted HCV follow-up (1\% vs. 9\%, $P<0.001$; Table 1). The reasons of incomplete HCV treatment were listed in Supplementary Table 1 . The SVR rate was significantly higher during the unre- 


\section{CLINCAL and MOLECULAF}

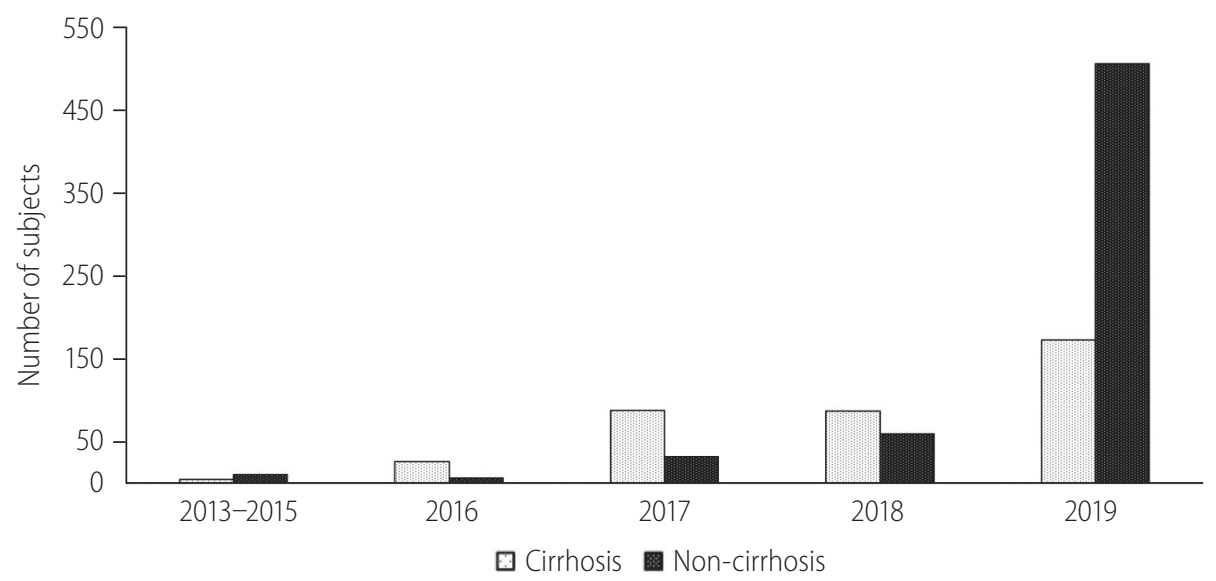

Figure 2. Exponential increase in non-cirrhotic hepatitis $C$ virus patients treated following unrestricted access to direct-acting antivirals.

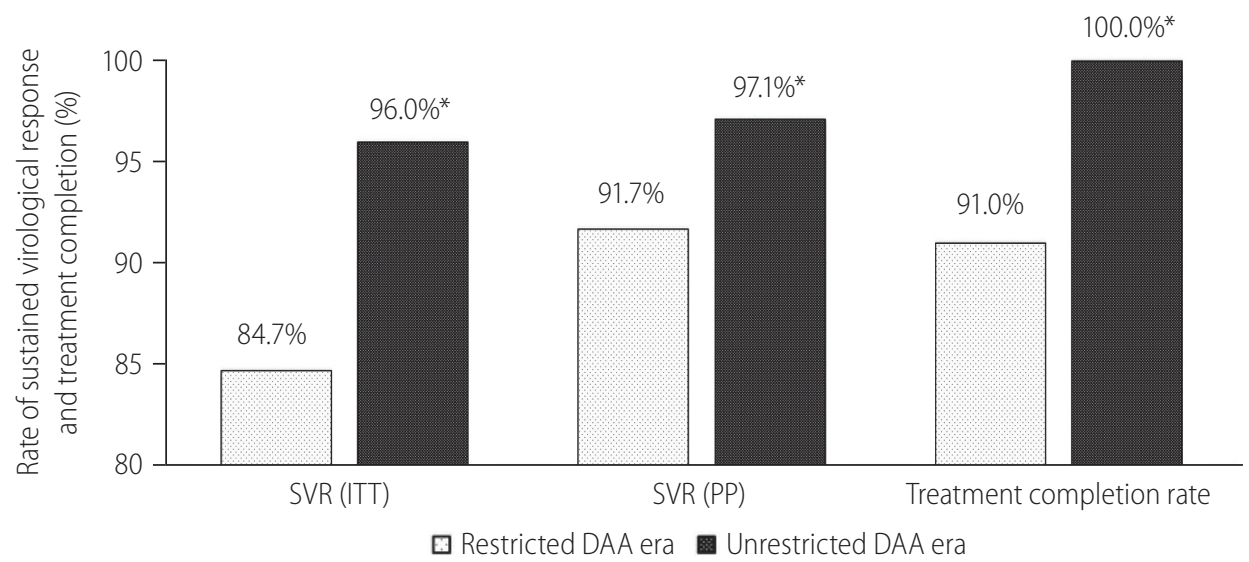

Figure 3. Treatment outcomes among incarcerated patients with liver cirrhosis. SVR, sustained-virological response; ITT, intention-to-treat; PP, per-protocol; DAA, direct-acting antivirals. ${ }^{*} P<0.05$ between restricted DAA and unrestricted DAA era.

stricted DAA access era than restricted DAA access era $(99.1 \%$ [95\% Cl, 98.1-99.7\%] vs. 92.1\% [95\% Cl, 87.4-96.8\%; $P<0.001$; Table 1).

With unrestricted DAA access, patients were treated at earlier stages (Table 1). These patients had higher serum albumin (45 vs. $43 \mathrm{~g} / \mathrm{L}, P<0.001)$, higher platelet count $\left(212 \times 10^{3} / \mathrm{uL}\right.$ vs. $168 \times 10^{3} / \mathrm{uL}$, $P<0.001)$ and lower serum bilirubin levels $(12.9 / \mathrm{mmol}$ vs. $15.7 / \mathrm{mmol}$, $P<0.001)$ when compared to HCV patients who were treated during restricted DAA access era.

\section{Subgroup analysis}

\section{Liver cirrhosis}

Among 383 cirrhotic HCV patients treated, 209 (54.6\%) were treated during the restricted DAA access era. Unrestricted access to DAA resulted in a relative reduction in the proportion of $\mathrm{HCV}$ cirrhosis treated ( $26 \%$ vs. $65 \%, P<0.001)$, primarily due to the exponential growth in non-cirrhotic HCV patients treated during unrestricted DAA era (Fig. 2). Subgroup analysis among HCV cirrhosis showed that more patients received DAA after unrestricted access to DAA ( $90 \%$ vs. $47 \%, P<0.001$; Supplementary Fig. 1). There were more patients completed treatment (100\% vs. 91\%, $P<0.001$; Fig. 3) and less patients defaulted treatment follow-up (7.7\% vs. 1.1\%, $P=0.002$; Supplementary Fig. 1). The SVR rate improved following unrestricted access to DAA $(97.1 \%[95 \% \mathrm{Cl}$, 93.3-99.1\%] vs. $91.7 \%$ [95\% Cl, 86.9-95.2\%]; $P=0.022$; Fig. 3).

\section{HCV GT3}

We performed subgroup analysis among 712 patients with GT3 HCV infection. Compared to non-GT3 patients, GT3 patients had 


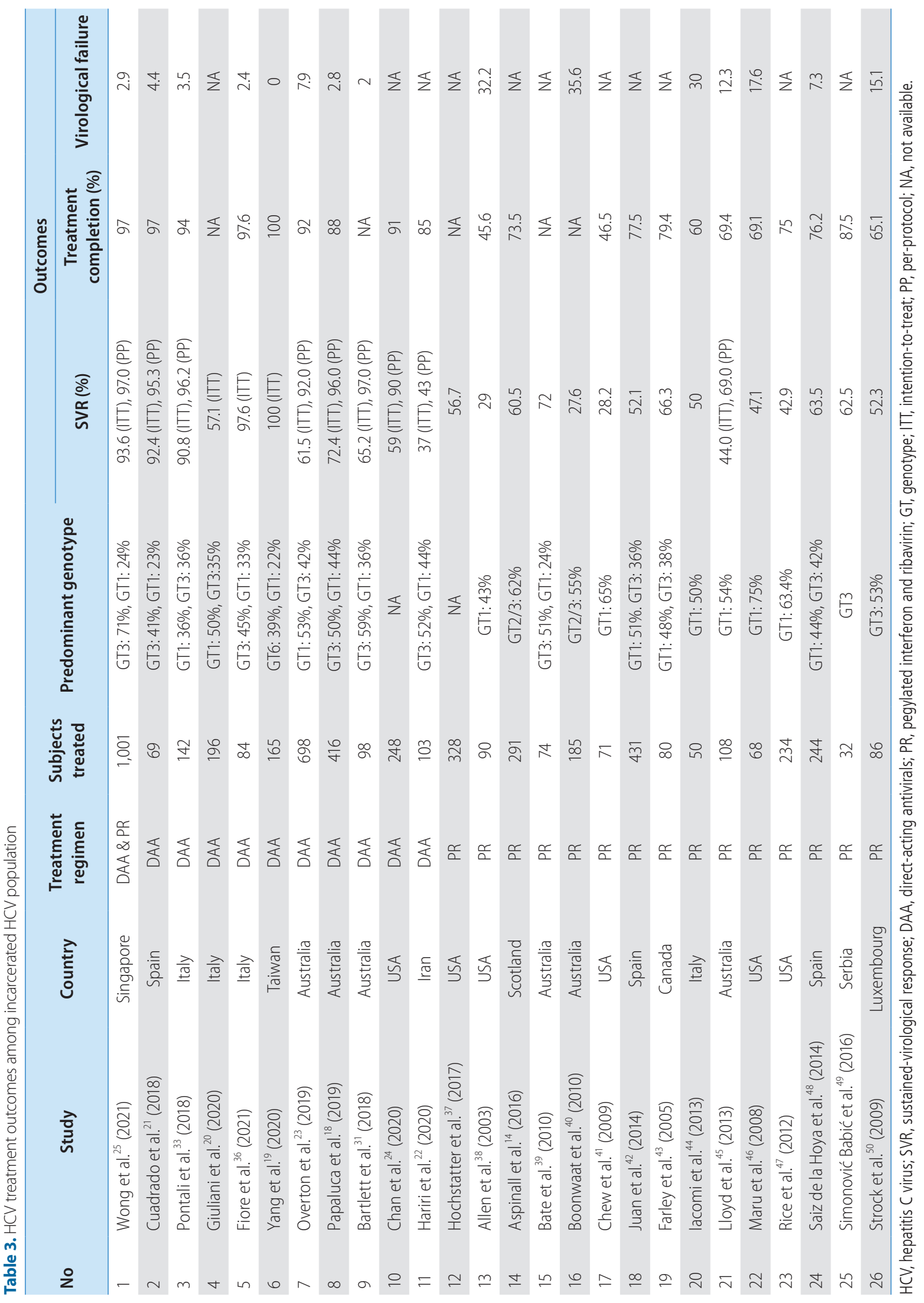


a higher proportion of liver cirrhosis (41.3\% vs. $30.8 \%, P=0.013$ ), lower serum albumin levels ( 44 vs. $45 \mathrm{~g} / \mathrm{L}, P=0.005$ ), lower platelet counts $\left(192 \times 10^{3} \mathrm{u} / \mathrm{L}\right.$ vs. $\left.213 \times 10^{3} \mathrm{u} / \mathrm{L}, P<0.001\right)$, higher serum bilirubin levels (14.2 vs. $12.7 \mathrm{mmol} / \mathrm{L}, P=0.007)$ and more prolonged prothrombin time (10.3 vs. 9.5 seconds, $P=0.018$ ). GT3 patients were less likely to received DAA (69.8\% vs. $98.6 \%$, $P<0.001)$ and had a lower SVR as compared to non-GT3 patients (96.1\% [95\% Cl, 94.3-97.3\%] vs. 99.3\% [95\% Cl, 97.4100.0\%]; $P=0.003$ ) based on PP analysis.

Subgroup analysis among GT3 HCV patients showed that more patients received DAA after unrestricted access to DAA (23.6\% vs. $92.8 \%, P<0.001$; Supplementary Fig. 2). There were more patients completed treatment ( $99 \%$ vs. $91 \%, P<0.001)$ and less patients defaulted treatment follow-up $(0.8 \%$ vs. $10.1 \%, P<0.001$; Supplementary Fig. 2). The SVR rates also improved following unrestricted access to DAA $(98.7 \%$ [95\% Cl, 97.2-99.5\%] vs. 90.1\% [95\% Cl, 85.3-93.8\%], $P<0.001)$.

\section{DAA vs. PR therapy}

Overall, 782 (78.1\%) HCV patients received DAA, while the remaining received PR therapy. The types of DAA used during the restricted and unrestricted DAA era were listed in Supplementary Table 2. Patients who received DAA were less likely to be cirrhotic ( $33 \%$ vs. $59 \%, P<0.001$ ) or had GT3 HCV infection ( $64 \%$ vs. $98 \%, P<0.001)$. As expected, DAA results in higher SVR rates than PR therapy (ITT: $97.6 \%[95 \% \mathrm{Cl}, 96.2-98.5 \%]$ vs. $79.5 \%$ [95\% Cl, 73.5-84.6\%], $P<0.001$; PP: $99.1 \%[95 \% \mathrm{Cl}, 98.1-$ $99.6 \%]$ vs. $88.8 \%[95 \% \mathrm{Cl}, 83.5-92.8 \%], P<0.001)$. Compared to PR therapy, patients treated with DAA treatment had a significantly higher treatment completion rates $(99.0 \%[95 \% \mathrm{Cl}, 98.0-$ 99.6\%] vs. $91.3 \%[95 \% \mathrm{Cl}, 86.8-94.7 \%], P<0.001)$ and a lower treatment default rates $(1.5 \%[95 \% \mathrm{Cl}, 0.8-2.7 \%]$ vs. $10.5 \%$ [95\% Cl, 6.8-15.3\%], $P<0.001$; Table 3).

Subgroup analysis among patients treated with DAA showed that unrestricted DAA access resulted in higher treatment completion rates $(99 \%$ vs. $97 \%, P=0.034)$ and lower treatment default rates $(0.8 \%$ vs. $5.2 \%, P=0.001)$. The overall SVR rate among patients treated with DAA increased following unrestricted access to DAA $(99.5 \%$ [95\% Cl, 98.6-99.9\%] vs. 96.9\% [95\% Cl, 85.393.8\%], $P<0.001)$.

\section{DISCUSSION}

The incarcerated HCV patients are a key population for HCV elimination. Given that the HCV population is relatively small in Singapore, immediate treatment scale-up among people who inject drugs will allow Singapore to achieved HCV elimination as per World Health Organization's target of an $80 \%$ reduction in incident cases, and a $65 \%$ reduction in mortality by $2030 .{ }^{29}$ While DAA is highly efficacious, the DAA treatment outcomes among incarcerated Asian HCV-infection patients remained scarce. We systematically reviewed and summarized the existing literatures on treatment outcomes among the incarcerated population in Table 3. We found that the treatment outcome among incarcerated population using DAA was encouraging with high SVR achieved among most cohorts based on the PP analysis. The treatment outcome of DAA among incarcerated Asian cohort was recently reported in two smaller studies from Taiwan and Iran. ${ }^{19,22}$ Yang et al. ${ }^{19}$ reported a high SVR of 100\% from 165 incarcerated patients with predominantly GT6 HCV infection. In contrast, Hariri reported a much lower SVR of 43\% among 103 incarcerated HCV patients who completed DAA treatment. ${ }^{22}$ In this large Asian cohort of 1,001 incarcerated HCV patients who were predominantly GT3 and followed up over 1,489 person-year, we demonstrated that unrestricted access to DAA resulted in rapid treatment scale-up, less treatment default and high SVR rates beyond $90 \%$.

Our study has three key findings. Firstly, the excellent HCV treatment outcomes among the incarcerated cohort highlight a unique opportunity for HCV elimination. Of note, this is the first largest study from South East Asia region reporting the treatment outcome of incarcerated HCV patients receiving both DAA and interferon-based therapy. Secondly, the unrestricted access DAA allows rapid scale-up to treat a large number of incarcerated HCV patients at early stages. A recent modelling study has demonstrated that a rapid treatment scale-up is crucial in order to achieve HCV elimination by $2030 .{ }^{29}$ Thirdly, high treatment success of DAA based on both the ITT and per protocol analysis can be achieved among incarcerated GT3 HCV patients in the realworld setting. We observed a continuous improvement in SVR, which corresponds to improving DAA access among this incarcerated cohort over the years (Table 2). To date, access to DAA remained an important barrier in many countries. Our findings highlight the vital role of universal access to DAA among incarceratedHCV population to rapidly scale-up our elimination effort in this key population in order to achieve the global goal of HCV elimination. 
Yu Jun Wong, et al. Unrestricted DAA access in incarcerated cohort

One important strength from our cohort is the high retention in our treatment cascade. Prior studies showed that treatment interruption as a result of early release or transfer can negatively impact the SVR rates among the incarcerated cohort. ${ }^{14}$ While high SVR beyond $90 \%$ is widely reported among incarcerated-HCV patients remained in follow-up, SVR by intention to treat was significantly lower, ranging between $37-72 \%{ }^{18,20,22-24}$ The difference is even more apparent among a larger cohort with high proportion of subjects being lost to follow-up. ${ }^{18,23,24}$ In this study, we successfully maintained a high retention rate in this large incarcerated cohort by ensuring adequate treatment duration, thus avoiding unplanned treatment interruption leading to treatment default. This is crucial because engaging patients who lost to follow up and their linkage of care after released remained challenging even in the DAA era.

Treatment access among incarcerated HCV patients is a global challenge. Because of limited prison budget and the exorbitant cost of DAA, only less than $1 \%$ of patients received treatment during incarceration. ${ }^{30}$ This has led to prioritizing HCV treatment based on the severity of liver disease as a common strategy in many countries. ${ }^{31,32}$ However, little is known about the impact of unrestricted access to DAA among the incarcerated population. Our study demonstrated that unrestricted access to DAA resulted in a rapid treatment scale-up and excellent treatment outcomes, thus making HCV micro-elimination among the incarcerated population an achievable goal in the real-world setting. ${ }^{32}$ Our findings were generalizable as high SVR rates have been demonstrated in other smaller studies among the incarcerated populations. ${ }^{21,33}$

The rates of HIV and HBV coinfection were low in our cohort (1.3\% and $1.3 \%$, respectively). Interestingly, a much higher rate of HIV and HBV coinfection was reported in a Taiwanese cohort at $4.7 \%$ and $8.9 \%$, respectively. We believe that our findings were representative as all patients were screened for both HIV and HBV upon the initiation of HCV treatment. In addition, our findings were also consistent with results of other published western cohorts. ${ }^{18,21}$

Despite international guidelines recommending unrestricted treatment for all HCV patients regardless of their incarceration status, DAA rationing is still common in many parts of the world due to financial and ethical concerns. ${ }^{23,24,34}$ Unrestricted HCV treatment represents a cost-effective strategy in the long run. However, affordability of HCV treatment must be addressed before unrestricted DAA access can be implemented. To improve the affordability of DAA, government can consider negotiating with manufacturers for a nominal price for DAA, or, adopt a prescrip- tion-based model for DAA. ${ }^{35}$ The unrestricted DAA access in Singapore is a successful example of price-negotiation with the manufacturer that resulted in price revision of SOF/VEL in 2018.

Universal access to DAA is a crucial step in HCV elimination in Singapore. Despite screening, improving linkage of care and education, only a small proportion of patients received HCV treatment. ${ }^{30}$ Even though more cirrhotic HCV patients were being treated during the restricted DAA era ( $79 \%$ vs. $50 \%, P=0.02)$, it is still insufficient to achieve HCV elimination in Singapore by 2030. Based on a recent modelling study, in order to achieve HCV elimination in Singapore by 2030, at least $630 \mathrm{HCV}$ patients has to be treated in $2019 .{ }^{26}$ A total of 681 patients was treated in 2019 alone following the unrestricted access to DAA, suggesting that HCV elimination by 2030 is indeed achievable in Singapore. In this study, we demonstrated that unrestricted access to DAA is an impactful strategy for a rapid treatment scale-up among incarcerated HCV patients.

Our findings should be interpreted within the limitations of retrospective studies. The number of subjects in earlier HCV care cascade and the total number of HCV patients between the two eras were not available in our study. We only included treated patients because we anticipated the impact of unrestricted DAA access to be more pronounced in this subgroup. Lastly, use of a newer pan-genotypic DAA might also contribute to a higher SVR during unrestricted DAA era.

In summary, unrestricted access to DAA is a key step in HCV elimination. We demonstrated that unrestricted access to DAA successfully removed the treatment barrier by allowing more HCV patients to be treated with DAA, at earlier stages, and resulted in a higher SVR and less treatment default. Our findings are generalizable and support unrestricted access to DAA as an impactful strategy for HCV micro-elimination among the incarcerated HCV patients in Singapore.

\section{Authors' contribution}

Concept: WYJ, PHT; Data collection: WYJ, SYC; Analysis: WYJ, PTH; Manuscript writing: WYJ, PTH, LNM, JT, FKM; Critical Reviewing: All

\section{Acknowledgements}

We would like to specially thank Dr John Hsiang, Dr Lim Kim Wei, Dr Chen Kaina, Mr Marcus Tong Zhi Jie, Ms Mai Lynn War and the department of Gastroenterology \& Hepatology, Changi General Hospital, for their contribution in this study. 


\section{Conflicts of Interest}

The authors have no conflicts to disclose.

\section{SUPPLEMENTARY MATERIAL}

Supplementary material is available at Clinical and Molecular Hepatology website (http://www.e-cmh.org).

\section{REFERENCES}

1. Yim HJ. Elimination of hepatitis C: what would be the practical approach? Clin Mol Hepatol 2021;27:97-99.

2. Huang CF, Yu ML. Unmet needs of chronic hepatitis C in the era of direct-acting antiviral therapy. Clin Mol Hepatol 2020;26:251-260.

3. Gallacher J, McPherson S. Progress towards micro-elimination of hepatitis C in the custodial setting. J Viral Hepat 2021;28:300-301.

4. Institute for Criminal Policy Research (ICPR). World prison population list. Twelve editions. ICPR web site, <https://www.prisonstudies.org/>. Accessed 21 Sep 2020.

5. Singapore Prison Service. Singapore Prison Service Annual Report 2019. Singapore Prison Service web site, <https://www.sps.gov. sg>. Accessed 21 Sep 2020.

6. Hsiang JC, Sinnaswami P, Lee MY, Zhang MM, Quek KE, Tan KH, et al. Point-of-care hepatitis $C$ screening with direct access referral to improve linkage of care among people with substance misuse: a pilot randomised study. Singapore Med J. 2020 Jul 30. doi: 10.11622/ smedj.2020116.

7. Moazen B, Saeedi Moghaddam S, Silbernagl MA, Lotfizadeh M, Bosworth RJ, Alammehrjerdi Z, et al. Prevalence of drug injection, sexual activity, tattooing, and piercing among prison inmates. Epidemiol Rev 2018;40:58-69.

8. Ruiz JD, Molitor F, Sun RK, Mikanda J, Facer M, Colford JM Jr, et al. Prevalence and correlates of hepatitis $C$ virus infection among inmates entering the California correctional system. West J Med 1999:170:156-160.

9. Stone J, Fraser H, Lim AG, Walker JG, Ward Z, MacGregor L, et al. Incarceration history and risk of HIV and hepatitis $C$ virus acquisition among people who inject drugs: a systematic review and metaanalysis. Lancet Infect Dis 2018;18:1397-1409.

10. Fox RK, Currie SL, Evans J, Wright TL, Tobler L, Phelps B, et al. Hepatitis $C$ virus infection among prisoners in the California state correctional system. Clin Infect Dis 2005;41:177-186.

11. World Health Organization. Guidelines for the Care and Treatment of persons diagnosed with chronic hepatitis C virus infection. Geneva: World Health Organization, 2018.
12. Lafferty L, Rance J, Grebely J, Lloyd AR, Dore GJ, Treloar C, et al. Understanding facilitators and barriers of direct-acting antiviral therapy for hepatitis C virus infection in prison. J Viral Hepat 2018;25:15261532.

13. Crowley D, Van Hout MC, Murphy C, Kelly E, Lambert JS, Cullen W. Hepatitis $C$ virus screening and treatment in Irish prisons from a governor and prison officer perspective - a qualitative exploration. Health Justice 2018;6:23.

14. Aspinall EJ, Mitchell W, Schofield J, Cairns A, Lamond S, Bramley $P$, et al. A matched comparison study of hepatitis $C$ treatment outcomes in the prison and community setting, and an analysis of the impact of prison release or transfer during therapy. J Viral Hepat 2016;23:1009-1016.

15. Enkelmann J, Gassowski M, Nielsen S, Wenz B, Roß S, Marcus U, et al. High prevalence of hepatitis $C$ virus infection and low level of awareness among people who recently started injecting drugs in a cross-sectional study in Germany, 2011-2014: missed opportunities for hepatitis C testing. Harm Reduct J 2020;17:7.

16. Wong YJ, Cheen MH, Hsiang JC, Kumar R, Tan J, Teo EK, et al. Economic evaluation of direct-acting antivirals for the treatment of genotype 3 hepatitis C infection in Singapore. JGH Open 2019;3:210216.

17. Agency for Care Effectiveness (ACE). Direct-acting antiviral agents for treating genotype 1 chronic hepatitis C. Technology guidance from the $\mathrm{MOH}$ drug advisory committee. ACE web site, <https:// www.ace-hta.gov.sg/docs/default-source/drug-guidances/directacting-antiviral-agents-for-treating-genotype-1-chronic-hepatitis-c(updated-1-oct-2020).pdf>. Accessed 20 Sep 2020.

18. Papaluca T, McDonald L, Craigie A, Gibson A, Desmond P, Wong $D$, et al. Outcomes of treatment for hepatitis $C$ in prisoners using a nurse-led, statewide model of care. J Hepatol 2019;70:839-846.

19. Yang TH, Fang YJ, Hsu SJ, Lee JY, Chiu MC, Yu JJ, et al. Microelimination of chronic hepatitis $C$ by universal screening plus directacting antivirals for incarcerated persons in Taiwan. Open Forum Infect Dis 2020;7:ofaa301.

20. Giuliani R, Casigliani V, Fornili M, Sebastiani T, Freo E, Arzilli G, et al. HCV micro-elimination in two prisons in Milan, Italy: a model of care. J Viral Hepat 2020;27:1444-1454.

21. Cuadrado A, Llerena S, Cobo C, Pallás JR, Mateo M, Cabezas J, et al. Microenvironment eradication of hepatitis $C$ : a novel treatment paradigm. Am J Gastroenterol 2018;113:1639-1648.

22. Hariri S, Sharafi $H$, Sheikh M, Merat S, Hashemi F, Azimian F, et al. Continuum of hepatitis $C$ care cascade in prison and following release in the direct-acting antivirals era. Harm Reduct J 2020;17:80.

23. Overton K, Clegg J, Pekin F, Wood J, McGrath C, Lloyd A, et al. Outcomes of a nurse-led model of care for hepatitis $C$ assessment and treatment with direct-acting antivirals in the custodial setting. Int J Drug Policy 2019;72:123-128. 
24. Chan J, Kaba F, Schwartz J, Bocour A, Akiyama MJ, Rosner Z, et al. The hepatitis $C$ virus care cascade in the New York City jail system during the direct acting antiviral treatment era, 2014-2017. EClinicalMedicine 2020;27:100567.

25. Wong YJ, Thurairajah PH, Kumar R, Tan J, Fock KM, Law NM, et al. Efficacy and safety of sofosbuvir/velpatasvir in a real-world chronic hepatitis C genotype 3 cohort. J Gastroenterol Hepatol 2021;36:1300-1308

26. The American Association for the Study of Liver Diseases (AASLD) and the Infectious Diseases Society of America (IDSA). Recommendations for testing, managing, and treating hepatitis C. AASLD and IDSA web site, <http://www.hcvguidelines.org>. Accessed 20 Sep 2020.

27. European Association for Study of Liver; Asociacion Latinoamericana para el Estudio del Higado. EASL-ALEH clinical practice guidelines: non-invasive tests for evaluation of liver disease severity and prognosis. J Hepatol 2015;63:237-264.

28. Talal AH, LaFleur J, Hoop R, Pandya P, Martin P, Jacobson I, et al. Absolute and relative contraindications to pegylated-interferon or ribavirin in the US general patient population with chronic hepatitis C: results from a US database of over 45000 HCV-infected, evaluated patients. Aliment Pharmacol Ther 2013;37:473-481.

29. Chaillon A, Thurairajah PH, Hsiang JC, Martin NK. What is required for achieving hepatitis $C$ virus elimination in Singapore? A modeling study. J Gastroenterol Hepatol 2021;36:1110-1117.

30. Beckman AL, Bilinski A, Boyko R, Camp GM, Wall AT, Lim JK, et al. New hepatitis $C$ drugs are very costly and unavailable to many state prisoners. Health Aff (Millwood) 2016;35:1893-1901.

31. Bartlett SR, Fox P, Cabatingan $H$, Jaros $A$, Gorton $C$, Lewis $R$, et al. Demonstration of near-elimination of hepatitis $C$ virus among a prison pulation: the lotus glen correctional centre hepatitis $C$ treatment project. Clin Infect Dis 2018;67:460-463.

32. Marshall AD, Pawlotsky JM, Lazarus JV, Aghemo A, Dore GJ, Grebely J. The removal of DAA restrictions in Europe - one step closer to eliminating HCV as a major public health threat. J Hepatol 2018;69:1188-1196.

33. Pontali E, Fiore $V$, lalungo AM, Ranieri R, Mollaretti $O$, Barbarini $G$, et al. Treatment with direct-acting antivirals in a multicenter cohort of HCV-infected inmates in Italy. Int J Drug Policy 2018;59:50-53.

34. Martin NK, Vickerman P, Dore GJ, Grebely J, Miners A, Cairns J, et al. Prioritization of HCV treatment in the direct-acting antiviral era: an economic evaluation. J Hepatol 2016;65:17-25.

35. Ocal S, Muir AJ. Addressing hepatitis $C$ in the American incarcerated population: strategies for Nationwide elimination. Curr HIVIAIDS Rep 2020;17:18-25

36. Fiore V, De Matteis G, Ranieri R, Saderi L, Pontali E, Muredda A, et al. HCV testing and treatment initiation in an Italian prison setting: a step-by-step model to micro-eliminate hepatitis C. Int J Drug
Policy 2021;90:103055.

37. Hochstatter KR, Stockman LJ, Holzmacher R, Greer J, Seal DW, Taylor QA, et al. The continuum of hepatitis $C$ care for criminal justice involved adults in the DAA era: a retrospective cohort study demonstrating limited treatment uptake and inconsistent linkage to community-based care. Health Justice 2017;5:10.

38. Allen SA, Spaulding AC, Osei AM, Taylor LE, Cabral AM, Rich JD. Treatment of chronic hepatitis $C$ in a state correctional facility. Ann Intern Med 2003;138:187-90.

39. Bate JP, Colman AJ, Frost PJ, Shaw DR, Harley HA. High prevalence of late relapse and reinfection in prisoners treated for chronic hepatitis C. J Gastroenterol Hepatol 2010;25:1276-1280.

40. Boonwaat L, Haber PS, Levy MH, Lloyd AR. Establishment of a successful assessment and treatment service for Australian prison inmates with chronic hepatitis C. Med J Aust 2010;192:496-500.

41. Chew KW, Allen SA, Taylor LE, Rich JD, Feller E. Treatment outcomes with pegylated interferon and ribavirin for male prisoners with chronic hepatitis C. J Clin Gastroenterol 2009;43:686-691.

42. Juan Jd, de la Hoya PS, Marco A, Antón JJ, Faraco I, Yllobre C, et al. Multicenter study on the discontinuation and efficacy of chronic hepatitis C treatment in the Spanish penitentiary population (EPIBAND study). Eur J Gastroenterol Hepatol 2014;26:1083-1089.

43. Farley J, Vasdev S, Fischer B, Haydon E, Rehm J, Farley TA. Feasibility and outcome of HCV treatment in a Canadian federal prison population. Am J Public Health 2005;95:1737-1739.

44. Iacomi F, Iannicelli G, Franceschini A, Migliorisi P, Rosati S, Piselli P, et al. HCV infected prisoners: should they be still considered a difficult to treat population? BMC Infect Dis 2013;13:374.

45. Lloyd AR, Clegg J, Lange J, Stevenson A, Post JJ, Lloyd D, et al. Safety and effectiveness of a nurse-led outreach program for assessment and treatment of chronic hepatitis $\mathrm{C}$ in the custodial setting. Clin Infect Dis 2013;56:1078-1084.

46. Maru DS, Bruce RD, Basu S, Altice FL. Clinical outcomes of hepatitis $C$ treatment in a prison setting: feasibility and effectiveness for challenging treatment populations. Clin Infect Dis 2008;47:952-961.

47. Rice JP, Burnett $D$, Tsotsis $H$, Lindstrom MJ, Cornett DD, Voermans $P$, et al. Comparison of hepatitis $C$ virus treatment between incarcerated and community patients. Hepatology 2012;56:1252-1260.

48. Saiz de la Hoya P, Portilla J, Marco A, García-Guerrero J, Faraco I, Antón J, et al. Directly observed therapy for chronic hepatitis C: a randomized clinical trial in the prison setting. Gastroenterol Hepatol 2014;37:443-451.

49. Simonović Babić J, Bojović K, Delić D, Katanić N, Mitrović N, Malinić J. Antiviral treatment of hepatitis $C$ in serbian prison setting: medical treatment outcomes and patients' adherence. Med Pregl 2016:69:85-91.

50. Strock P, Mossong J, Hawotte K, Arendt V. Access to treatment of hepatitis C in prison inmates. Dig Dis Sci 2009;54:1325-1330. 